\title{
Natural Killer Cell Leukemia: Diagnosis, Pathogenesis and Treatment
}

\author{
Shoko Kobayashi \\ Kohseichuo Hospital \\ Japan
}

\section{Introduction}

Natural Killer(NK) cell nenoplasm is heterogeneous disease group. In the latest World Health Organization(WHO) classification of tumors of hematopoietic and lymphoid tissue(2008), disease entities considered as NK cell deviation are 1) Aggressive NK cell leukemia (ANKL), 2) NK cell lymphoblastic leukemia/lymphoma, 3) Extranodal NK/T-cell lymphoma, nasal. While several NK related diseases are proposed and investigated actively, optical treatment of NK cell neoplasms remains uncertain. However, several new chemoagents and transplantation are now in progress.

\section{Natural killer cell}

\subsection{Definition}

Natural killer(NK) cells are the first lymphoid cells deployed in the defense against tumors and viral infection(Sun, 2010). Their activity is regulated by the interplay between inhibitory receptors, most of which recognize MHC class I molecules on target cells, and activating receptors which bind various ligands. Because NK cells originate from progenitor cells that can give rise to either NK or T cells; NK cells are phenotypically and immunologically similar to T cells. NK cells express T cell-associated markers, including CD2, cytoplasmic CD3(cCD3), CD7 and CD8, subsets of cytotoxic T cells and also express the NK-associated markers, CD16, CD56 and CD57. NK cells do not rearrange the T-cell receptor (TCR) genes. Early in development, NK-cell progenitors express no specific markers. Some markers that might be considered relatively more specific with NK progenitors, such as CD94 or CD161 are no commonly tested.

Important inhibitory receptors are members of the immunoglobulin-like receptor (KIR) family as well as CD94/NKG2 heterodimers. Examples for activating receptors are NKG2D (CD314), DNAM-1 (CD226) and the well-characterized natural cytotoxicity receptors (NCRs), NKp30(CD337), NKp44 (CD336) and NKp46 (CD335). Activating receptors such as NKG2D, recognize ligands that are not usually expressed by healthy cells but rather expressed by infected cells. The inhibitory receptors of NK cells interact with specific MHC class I molecules; for example, CD94/NKG2 binds to HLA-E, and KIR binds to HLA-B. The major constituents of cytotoxic granules are perforin and granzyme B. 


\subsection{Development}

Because NK cells exhibit considerable immunophenotypic similarity to $\mathrm{T}$ cells, they are thought to originate from a bipotent NK/T-progenitor cell. Moreover, human CD34(+) hematopoietic progenitors develop into NK cells in vitro in the presence of cytokines, hydrocortisone and stromal cells through the recruitment of myeloid precursors(Grzywacz, 2011). Cells at more advanced stages of myeloid differentiation (those with higher levels of CD13 and macrophage colony-stimulating factor receptor [M-CSFR]) could also differentiate into NK cells in the presence of cytokines (interleukin-7, interleukin-15, stem cell factor, and fms-like tyrosine kinase-3 ligand), stromal cells, and hydrocortisone. NK cells derived from myeloid precursors (CD56(-)CD117(+)M-CSFR(+)) showed more expression of KIR.

\section{Natural killer cell neoplasm}

\subsection{Diagnosis}

Accurate diagnosis of NK-cell neoplasm requires analysis of clinical presentation and of cell/tumor morphology, immunophenotype, and genotype(Oshimi, 2007). Expression of at lease one NK cell marker(CD56, CD16, or CD57), lack of expression of surface CD3, B-cell antigen(CD19, ad CD20) and MPO and other lineage markers and/or TCR Ig genes in germline configuraion in tumors are diagnostic factors. During the early stages of NK cell neoplasm, tumor cells do not express any tumor-specific markers or they express markers also found in T cell acute lymphoblastic leukemia (ALL), including CD7, CD2, CD5, and cCD3. Therefore it is difficult to distinguish between T- cell ALL and NK-cell tumors may be difficult. Later-stage but more specific markers, such as CD16, are rarely expressed in NK cell leukemia while some markers, such as CD94 and CD161, that might be considered relatively more specific expressed on NK progenitors. However, even now, it is difficult for us to distinguish NK cell neoplasm from myeloid neoplasms and T-cell neoplasms. It is hoped that wider availability of more specific NK markers including panels of antibodies against KIRs.

\subsection{Classification}

In the latest World Health Organization(WHO) classification of tumors of hematopoietic and lymphoid tissues(2008), acute leukemia with phenotype of NK cells includes(Swerdlow, 2008); 1) Aggressive NK cell leukemia (ANKL) and 2) NK cell lymphoblastic leukemia/lymphoma. Other NK cell neoplasm and related disease are reported in literature; 1) extranodal NK/T-cell lymphoma, nasal, 2) Myeloid/NK leukemia, and 3) Other CD56positive neoplasm(Oshimi, 2007).

\subsubsection{Aggressive NK cell leukemia (ANKL)}

ANKL is a catastrophic disease(Suzuki, 2010; Ham, 2010). ANKL has no gender preference, with the median age of presentation being in the third decade. ANKL is more prevalent among Asians than other ethnic populations. Circulation neoplastic cells varying morphologically form large granular lymphocytes to frank blast, may be present despite treatment. The neoplastic cells are CD2+, surface CD3-, CD3e+, CD56+, and positive for cytotoxic molecules. Immunophenoytpe is identical to that of extranodal type is identical to that of extranodal NK/T-cell lymphoma, except that CD16 is frequently positive. Patients have generally only been unwell for a few weeks, presenting with significant weight loss, 
jaundice and a high fever. Skin infiltration, lymphoadenopaty and hepatosplenomegaly are common. Severe anemia and throm Survival measured in some weeks. It is unclear whether aggressive NK-cell leukemia represents the leukemic counterpart of extranodal NK/T-cell lymphoma. Strong association with Epstein-Barr virus(EBV) suggests a mechanism of pathogenesis.

\subsubsection{NK cell lymphoblastic leukemia/lymphoma}

This neoplasm has been very difficult to define, because of considerable confusion in the literature(Swerdlow, 2008). WHO proposed that the diagnosis of precursor NK lymphoblastic leukemia/lymphoma may be considered in a case that expresses CD56 along with immature $\mathrm{T}$ cell-associated markers such as CD7, CD2 or even including cCD3, provided the tumor lacks B-cell and myeloid markers, and that theT cell and Ig receptor genes are in the germline configuration. Clinical features associated with these NK neoplasms are not well known. Previously we reported a 69-year-old man with NK cell lymphoblastic leukemia/lymphoma(Kobayashi, 2010). He presented with skin and bone marrow involvement upon initial diagnosis. Neoplastic cells were blastic in appearance with a CD3-, CD4-, CD8-, CD7-, CD16-, CD56+ and HLA-DR+ phenotype. Molecular studies showed germline configuration of both immunoglobulin $\mathrm{H}$ and $\mathrm{T}$ cell receptor genes, and negative results for Epstein-Barr virus-encoded small RNA (EBER).

Optimal treatment of this disease has not been determined. The presence or absence of Pglycoprotein $(\mathrm{P}$-gp) on tumor cells is one factor that influences the choice of anti-cancer drugs(Egashira, 1999).

\subsubsection{Extranodal NK/T-cell lymphoma, nasal}

Extranodal NK cell lymphomas(ENKL) are more prevalent in Asia, Mexico, and Central and South America and are characterized by extranodal presentation and an aggressive clinical course(Schmitt, 2011; Yok-Lam, 2011; Suzuki, 2009; Ng, 2011). Two types of ENKL (nasal and extranasal ENKL) were reported. Because they share the same histology, the WHO classification groups both nasal and extranasal ENKL in the same category. However, nasal and extranasal ENKL have different clinical manifestations, treatment approaches and prognosis.

International Peripheral T-cell lymphoma Project showed the clinical characteristics of both NK cell lymphoma among 1153 new adult cases of peripheral/T-cell lymphoma at 22 centers in 13 countries(Au, 2009). 136 cases (11.8\%) of extranodal NK/T-cell lymphoma were identified (nasal 68\%, extranasal 26\%, aggressive/unclassifiable 6\%). There were no differences in age, sex, ethnicity, or immunophenotypic profile between the nasal and extranasal cases, but the latter had more adverse clinical features. The median overall survival (OS) was better in nasal compared with the extranasal cases in early- (2.96 vs 0.36 years) and late-stage disease ( 0.8 vs 0.28 years). Among nasal cases, both the International Prognostic Index and Korean NK/T-cell Prognostic Index were prognostic. In addition, Ki67 proliferation greater than $50 \%$, transformed tumor cells greater than $40 \%$, elevated Creactive protein level $(\mathrm{CRP})$, anemia $(<11 \mathrm{~g} / \mathrm{dL})$ and thrombocytopenia $(<150 \times 10(9) / \mathrm{L})$ predicts poorer OS for nasal disease. No histologic or clinical feature was predictive in extranasal disease.

The strong association of EBV was observed, with type II latency pattern(EBNA-1+, EBNA2+, LMP1+), and commonly showed a 30-base pair deletion in the latent membrane protein- 
1(LMP-1) gene(Swerdlow, 2008). Genome-wide gene expression profiling of extranodal NK/T lymphoma(NKTL) showed genes differentially expressed between NKTL and normal NK cells revealed significant enrichment for cell cycle-related genes and pathways, such as PLK1, CDK1, Aurora-A, activation of Myc and nuclear factor kappa B (NF-kB), and deregulation of p53. A significant percentage of NKTLs $(n=33)$ overexpressed c-Myc (45.4\%), p53 (87.9\%), NF-kB p50 (67.7\%) and survivin(97\%)(Huang, 2010). It is possible to propose a model of NKTL pathogenesis where deregulation of p53 together with activation of Myc and NF-KB, possibly driven by EBV LMP-1, results in the cumulative up-regulation of survivin. Down-regulation of survivin with Terameprocol (EM-1421, a survivin inhibitor) results in reduced cell viability and increased apoptosis in tumour cells, suggesting that survivin may be a potential oncoregulative gene in NKTL. Overexpressed PDGFRA, deregulation of the AKT, JAK-STAT, and nuclear factor-kappaB, RelA and recurrent copy number aberrations (AKT3 [1q44], IL6R [1q21.3], CCL2 [17q12], TNFRSF21 [6p12.3]) was reported $(\mathrm{Ng}, 2011)$.

The common cytogenetic abnormality of ENKL is del(6)(q21q25)or i(6)(p10). Integrative analysis also evidenced deregulation of the tumor suppressor HACE1 in the frequently deleted 6q21 region(Zhang, 2007). HACE1 is novel E3 ubiquitin ligase, frequently downregulated in human tumors. Genetic inactivation of HACE1 in mice results in the development of spontaneous, late-onset cancer. A second hit from either environmental triggers or genetic heterozygosity of another tumor suppressor, p53, markedly increased tumor incidence in a Hace1-deficient background. Re-expression of HACE1 in human tumor cells directly abrogates in vitro and in vivo tumor growth, whereas downregulation of HACE1 via siRNA allows non-tumorigenic human cells to form tumors in vivo. Mechanistically, the tumor-suppressor function of HACE1 is dependent on its E3 ligase activity and HACE1 controls adhesion-dependent growth and cell cycle progression during cell stress through degradation of cyclin D1.

\subsubsection{Myeloid/NK leukemia}

The entity of myeloid/NK-cell acute leukemia may be of precursor NK origin, however myeloid/NK-cell acute leukemia cannot be distinguished form acute myeloid leukemia with minimal differentiation(Tang, 2008). Reportedly, myeloid/NK cell precursor acute leukemia (MNKPL) give rise to blasts that are cytochemically myeloperoxidase negative $(\mathrm{MPO}(-))$ and phenotypically CD56(+)CD3(-)CD7(+)CD34(+) and have myeloid antigens(Guan, 2011). In contrast, myeloid/NK cell acute leukemia (MNKL) give rise to blasts that are cytochemically MPO(dim) and phenotypically CD56(+), CD16(-), CD3(-), CD)33(+)HLA-DR(-). Several cases of childhood NK leukemia, four MNKPL and one MNKL, were reported in China. The extramedullary involvement that usually occurs in cases of adult MNKPL was not observed in these cases of childhood MNKPL. Those with MNKPL were treated using a protocol designed for childhood high-risk ALL containing cytarabine, mitoxantrone, etoposide, l-asparaginase, and methotrexate depending on the myeloid and lymphoid characteristics of the MNKPL. They responded slowly to chemotherapy and were in complete remission (CR), except one who died from pneumonia while in CR. Therefore, protocols that combine agents used against acute myeloid leukemia with agents used against ALL are apparently effective against childhood MNKPL.

A 6-year-old Japanese child was reported to be myeloid/NK cell precursor acute leukemia (MNKL)(Shiba 2010). The patient was treated by cord blood transplantation from an HLA 1- 
locus mismatched unrelated donor after chemotherapy comprising cytosine arabinoside, idarubicin, etoposide, and L-asparaginase. The nonsense mutation, C7412A, resulting in S2471X, where X is a terminal codon, in the PEST domain of NOTCH1 were observed, suggesting a possible role in the leukemogenesis of MNKL.

In addition, CD7+ and CD56+ myeloid/natural killer cell precursor acute leukemia was proposed(Suzuki, 1997; Suzuki, 2005, Piichowska, 2007). Striking extramedullary involvement was evident at initial presentation, with peripheral lymphadenopathy and/or mediastinal masses. Expression of CD7, CD33, CD34, CD56, and frequently HLA-DR, but not NK, T-cell, and B-cell markers was observed. Myeloperoxidase was negative. Almost all presented germline configurations of the TCR $\beta$ and $\gamma$ chain genes and Ig heavy chain gene. Despite intensive chemotherapy, including allogeneic bone marrow transplantation, most persued fatal courses within 41 months. Forty-nine patients with CD7(+) CD56(+) acute myeloid leukemia were analyzed(Suzuki, 1997). There were 17 patients with a classification of M0, which corresponded to MNKPL, and 32 patients with an AML classification other than M0 (i.e., M1-M7; 9 each for M1 and M2, 1 for M3, 3 for M4, 4 for M5, and 6 for M7). The age distribution was similar in both groups, but the MNKPL group, i.e., CD7(+) CD56(+) M0, had significantly more males than the CD7(+) CD56(+) M1-M7 group. The disease localization and the hematological manifestations were different between the groups; specifically, the patients with MNKPL presented with lower white blood cell counts and fewer circulating leukemic blasts, less anemia, less thrombocytopenia, and more frequent extramedullary involvement. The prognosis was poor in both groups. These findings suggest that extramedullary involvement of myeloid/NK cell precursor acute leukemia is not directly derived from the presence of CD7 and CD56 antigens on leukemic cells. The poor prognosis of CD7(+) CD56(+) M1-M7 suggests that this phenotype may act as a prognostic factor for AML, but this assertion should be confirmed in further clarification.

\subsubsection{Other CD56 positive neoplasm}

CD56 has been recognized as a sensitive marker for NK cells and has become popular for identifying NK cell neoplasms. Approximately 200 CD56+ hematopoietic neoplasms with immature features have been reported in the literature under different names. It is difficult to determine whether these tumors are of a truly derived from NK cells. For example, tumors that were previously defined as "blastic NK lymphoma" are now classified into two entities: 1) blastic plasmacytoid dendritic cell neoplasm; and 2) NK cell lymphoblastic leukemia/lymphoma(Pilichowska, 2007). The identification of the cell type, blastic plasmacytoid dendritic cell led to the definition of a new disease entity of blastic plasmacytoid dendritic cell neoplasm.

Recently a new disease category, NK-cell enteropathy, was proposed based eight patients with atypical NK-cell lymphoproliferative lesions that mimicked NK- or T-cell lymphoma(Mansoor, 2011). These patients presented with vague gastrointestinal symptoms and with lesions involving stomach, duodenum, small intestine, and colon. Biopsies revealed a mucosal infiltrate of atypical cells with an NK-cell phenotype (CD56(+)/TIA$1(+)$ /Granzyme $\mathrm{B}(+) / \mathrm{cCD} 3(+))$.EBER was negative, and TCR-ץ gene rearrangement showed no evidence of a clonal process. Some patients received aggressive chemotherapy followed by auto HSCT. Five patients were followed without treatment. However, no patient developed progressive disease or died of lymphoma. 
While a distinct disease entity, "lymphomatoid gastropahty (LyGa)", was proposed based on the analysis of 10 other patients with unrecognized self-limited NK-cell proliferation in the stomach(Takeuchi, 2010). Endoscopy showed elevated lesion(s). The cells were CD2+/-, $\mathrm{sCD}^{-}, \mathrm{cCD}^{+}, \mathrm{CD}^{-}, \mathrm{CD}^{-}, \mathrm{CD}^{+}, \mathrm{CD}^{-}, \mathrm{CD}^{-} 6^{-}, \mathrm{CD}^{-} 0^{-}, \mathrm{CD}^{+} 5^{+}, \mathrm{CD}^{+} 6^{+}, \mathrm{CD}_{117}-, \mathrm{CD} 158 \mathrm{a}^{-}$, CD161-, $\mathrm{T}$ cell-restricted intracellular antigen- $\mathbf{1}^{+}$, granzyme $\mathrm{B}^{+}$, perforin ${ }^{+}$, Epstein-Barr early $\mathrm{RNA}^{-}, \mathrm{T}-\mathrm{CR} \alpha \beta^{-}$, and TCR $\gamma \delta^{-}$. Most lesions underwent self-regression. Three cases relapsed, but none of the patients died. LyGa is proposed as a pseudomalignant process because of its clinical characteristics.

\section{Pathogenesis}

\subsection{EBV}

NK cell neoplasms are strongly related with latent EBV infection.

EBV is a ubiquitous human gamma-herpesvirus that preferentially establishes latent infection in viral infected B-lymphocytes(Pizzigallo, 2010). The structure of EBV shows a linear double-stranded DNA, an capside, 162 capsomers and an envelope. The EBV genome encodes nearly 100 viral proteins. During viral replication, these proteins play a fundamental role in regulating the expression of viral genes, replicating viral DNA, forming structural components of the virion, modulating the host immune response, and forcing these cells immortal. The receptor for the virus on epithelial cells and B lymphocytes is the CD21 molecule, which is also the receptor for the C3d component of complement. The major histocompatibility complex (MHC) class II molecule serves as cofactor for the infection of B cells.

Cellular infection from EBV could have two possible outcomes. A lytic infection occurs wherein virions are produced and the host cell is lysed. Lytic infection typically occurs in epithelial cells and partly in plasma cells. EBV may induce a latent infection by generating an episome, the circular EBV genome, that is located in the nucleus of host lymphocytes.

The response of CD8-positive $(\mathrm{CD} 8+)$ cytotoxic T-cells is crucial to control the primary infection, and these cells show a predominant role in infectious mononucleosis(IM), being present in the circulation and tissues in very high numbers. These cells probably give rise to most of the symptoms and signs of IM as a result of massive production of cytokines, including lymphotoxin, tumor necrosis factor- $\alpha$, interleukin (IL)- $1 \beta$ and IL-6. That CD8+ T cells are essential for recovery from IM is exemplified by the consequence of primary EBV infection.

The latent viral infections in which the virus persists in a form unable to be identified through standard methods. The mechanisms of EBV latency have been carefully examined both because they represent the virus strategy to elude the response of the immune system of the host, and because they are correlated with those oncologic conditions associated to the viral persistence, particularly lymphoma and lymphoproliferative disorders. During latency, resting memory B cells represent the site of persistence of EBV within the body. In normal adults, from 1 to $50 \mathrm{~B}$ cells per million in the circulation are infected with EBV, and the number of latently infected cells remains stable over years.

There are different programs of EBV latency that are probably associated with different viral strategies aimed at surviving host immunological responses, and some of these programs have been correlated with malignancies typically associated with viral persistence. Four latency programs was proposed(Table I, II). In the first form, only EBNA-1 and EBER are expressed, whereas in the second form EBNA-1, LMP-1, LMP-2, and EBER are expressed. In 
the third pattern, all the latency genes are expressed. A fourth pattern of latency is seen in B cells obtained from the peripheral blood of healthy persons infected with EBV in the past, in which only EBER and LMP-2, and in some studies, EBNA-1 RNA have been detected. Burkitt's lymphoma is characterized by the type 1 of the latency program, the nasopharyngeal carcinoma, Hodgkin's lymphoma and peripheral $\mathrm{T}$ cell lymphoma by the type 2 . The type 3 of the latency program should characterize other lymphoproliferative diseases induced by EBV in immunocompromised hosts as well as the IM. These mechanisms are involved virological factors associated with EBV antigenic characteristics, host factors with particular regard to the genetic and immune systems and environmental co-factors. Of the nearly 100 viral genes that are expressed during replication, only 10 are expressed in latently infected B cells in vitro: two types of nontranslated RNA, six nuclear protein and two membrane proteins. Among several viral proteins, EBNA1 and LMP1 are important in tumorigenesis.

\subsubsection{EBV nuclear antigen 1 (EBNA-1)}

EBV latent infection, and its associated oncogenic potential, is dependent on genome maintenance functions of EBV nuclear antigen 1 (EBNA-1), one of six EBNAs expressed from a common promoter(Wp and then $\mathrm{Cp}$ ) upon infection of naive B cells. EBNA1 initiates latent viral replication in $B$ cells maintains the viral genome copy number, and regulates transcription of to the EBV-encoded latent genes. These activity are mediated through the ability of EBNA1 to bind viral-DNA.

Resistance to apoptosis is an important in tumorigenic process. EBNA1 forms a complex with Sp1 or Sp1-like proteins bound to their cis-element at the survivin promoter. This interaction enhances the activity of the complex and up-regulates survivin. Knockdown of survivin and EBNA1 showed enhanced apoptosis in EBV -infected cells and thus supports a role for EBNA1 in suppressing apoptosis in EBV-infected cells(Ian, 2008).

Cell proliferation was inhibited in an EBV-positive ENKL cell line by RNA interference (RNAi)-mediated silencing of EBNA1. Silencing of EBNA1 expression by RNAi inhibited cell growth, increased the expression of the p27 protein, and caused cell cycle arrest in $G(1)$.

\begin{tabular}{|c|c|c|}
\hline Gene & Protein & Function \\
\hline BKRF1 & EBNA-1 & $\begin{array}{c}\text { viral genome maintenance } \\
\text { transcription of EBV latent genes } \\
\end{array}$ \\
\hline BKRF1 & EBNA-2 cell immortalization \\
\hline BLF-BERF & EBNA-3 & B cell immortalization \\
\hline BNLF1 & LMP-1 & B cell immortalization \\
\hline BNLF2 & LMP-2 & oncoprotein \\
\hline BCRF1 & EBER1,2 & prevents cell lytic-cycle entry \\
\hline BARF0 & Bam HIA transcripts & regulates PKC activity \\
\hline
\end{tabular}

Table 1. EBV latent viral genes 


\subsubsection{Latent membrane protein-1(LMP-1)}

EBV encodes a viral oncogene, LMP1 (latent membrane protein-1). LMP1 is expressed in EBV-associated lymphoma and is essential for B-cell transformation and for disruption of cellular signal transduction(Dellis, 2009). LMP1 acts by constitutive activating multiple signaling pathway, including the NK-kB pathway. In extranodal NK/T-cell lymphoma, nasal type, deletion of $30 \mathrm{bp}$ in the LMP-1 gene are reported, but the molecular and pathogenic significance of these deletions is not clear.

\begin{tabular}{|l|c|c|c|c|c|c|c|}
\hline & EBNA-1 & EBNA-2 & EBNA-3 & LMP-1 & LMP-2 & EBER & Disease \\
\hline Type 1 & + & - & - & - & - & + & Burkitt's lymphoma \\
\hline Type 2 & + & - & - & + & + & + & $\begin{array}{c}\text { Nasopharyngea carcinoma } \\
\text { Hodgkin's disease }\end{array}$ \\
\hline Type 3 & + & + & + & + & + & + & $\begin{array}{c}\text { Lymphoproliferative disease } \\
\text { Infectious mononucleosis }\end{array}$ \\
\hline others & + & - & - & - & + & + & Healthy carieer \\
\hline
\end{tabular}

Table 2. Expression of EBV latent genes in disease

\subsection{Cytogenetics}

Several cytogenetic abnormalities have been reported in ENKL cases(Schmitt, 2011).

Reported abnormal chromosomal gains are 1q21-q44, 2q13-q14, 2q31.1-q32.2, 6p25-p11.1, 7q11.2-q34, 7q35-q36, 17q21.1, 20pter-qter • while abnormal chromosomal losses are 6q16q25, 11q23.1, 11q24-q25, 13q14.11, 17p13.3

The genes for responsible for above abnormalities have been investigated and are speculated to be EBV LMP2-TR; survival/apoptosis related: CCND3, SERPINB9, FASL, or TNFAIP3; angiogenesis related: HGFR (MET), VEGFR2, VEGFA, or HIF1a; cell signaling pathway related: JAK-STAT, AKT, NOTCH, WNT, or PDGF; tumor-suppressor gene related: del6q21(PRDM1, ATG5, AIM1, or HACE1); and other pathways/genes: TP53, TP73, or AURKA.

\section{Staging}

A standard staging system for NK-T-cell lymphomas is lacking. Moreover, the Ann-Arbor staging system is probably unsatisfactory for NK/T-cell lymphomas.

Involvement of bone marrow $(\mathrm{BM})$ is an important staging and prognostic factor(Huang, 2005). A study was done to evaluate the use of EBER -1 in situ hybridization (EBER-1 ISH) to detect occult micrometastasis in the bone marrow (BM) of patients with nasal NK/Tcell lymphoma. Conventional morphologic examinations failed to identify any lymphoma involvement in the BM specimens obtained at initial staging. However, some BM specimens were positive for EBER-1. A lower survival rate was seen in patients with BM positive for EBER-1-positive BM specimens, and only the BM EBER-1 ISH result was shown to be an independent variable predicting overall survival in stage I / II patients, suggesting that EBER-1 positivity in BM is the major determinant of a poor prognosis. These findings indicate that EBER-1-positive BM specimens are an important indicator of a poor prognosis, and based on this study, BM EBER-1 ISH evaluation is recommended for accurate staging. 


\section{Prognosis; Prognostic factors}

To improve the stratification of patients for treatment, some prognostic models have been applied to NK-cell neoplasms.

The International Prognostic Index(IPI), taking into account the stage, age, performance status, number of extranodal sites and the LDH level, is evidently useful for assess the prognosis of patients with NK-cell lymphoma(Lee, 2006).

Clinical features and prognosis of 22 with ANKL and 150 with ENKL patients was reviewed(Suzuki, 2010). The ENKLs consisted of 123 nasal and 27 extranasal (16 cutaneous, 9 hepatosplenic, 1 intestinal and 1 nodal) lymphomas. Multivariate analysis showed that four factors (non-nasal type, stage, performance status and numbers of extranodal involvement) were significant prognostic factors. Using these four variables, an NK prognostic index was successfully constructed. Four-year overall survival of patients with zero, one, two and three or four adverse factors were $55 \%, 33 \%, 15 \%$ and $6 \%$, respectively.

Korean prognostic index (K-IPI) was analyzed as a prognostic model specifically for ENKL(Lee, 2006). Prognostic factors for survival were "B" symptoms, stage, LDH level, and regional lymph nodes. K-IPI identified four different risk groups: group 1, no adverse factor; group 2, one factor; group 3, two factors; and group 4, three or four factors. The K-IPI showed a superior prognostic discrimination when compared with the IPI. Notably, when the K-IPI system was used, $7 \%$ of patients were in group $1 ; 31 \%$ were in group $2 ; 20 \%$ were in group 3 , and $22 \%$ were in group 4 ; in contrast, $81 \%$ of patients were categorized as low or low-intermediate risks using IPI.

In nasal NK/T-cell lymphoma, measurement of circulating plasma EBV DNA is reportedly useful for evaluating the prognosis of patients(Hsieh, 2007).

\section{Treatment}

1 Chemotherapy
a) L-asparaginase
b) Methotrexate
c) combination regimen SMILE

2 Hematopoietic stem cell transplantation(HSCT)

3 New therapeutic strategies
a) Bortezomib
b) Pegasparaginase
c) Kinase inhibitor
d) Antibody treatment

4 Radiation

Table 3. Treatment of NK neoplasm 


\subsection{Chemotherapy}

\subsubsection{L-asparaginase containing regimen}

The poor treatment results of conventional chemotherapy reatments for NK cell neoplasm are often attributed to the high expression level of the drug-exporting protein Pglycoprotein, which results in the multidrug-resistance(MDR) phenotype(Jaccard, 2009). Lasparaginase(L-asp), an enzyme that hydrolyzes serum L-asp, induces asparagine starvation of tumors with low expression of asparagine synthetase. This results in rapid inhibition of protein synthesis and delayed inhibition of DNA and RNA synthesis in lymphocytes.

Thirty-three Chinese patients with midline NK/T-cell lymphoma nasal-type were received L-asp based salvage regimen for 2 approximately 6 cycles (median 3 cycles) plus locoregional radiation. Seventeen of the $33 \mathrm{CHOP}$ failures (51.5\%) (L-asp group) reached CR(Yong, 2006). The 5-year overall survival (OS) rates were $55.9 \%$ for L-asp group. On univariate analysis, disease stage, fever symptom and performance status were significant factors influencing overall survival. On multivariate analysis, only disease stage and fever symptom remained as independently significant factors influencing OS.

The multicentric French retrospective study of 15 patients with relapsed, refractory, or disseminated disease, treated with L-asp - containing regimens(Jaccard, 2009). All but two of the patients had an objective response to L-asp-based treatment. Seven patients reached complete remission and only two relapsed. These data confirm the excellent activity of Lasp-containing regimens in refractory extranodal NK/T-cell lymphoma and also aggressive NK-cell leukemia. The results indicated that L-asp based regimen might be a promising new salvage or even first-line chemotherapeutic regimen for NK-cell neoplasms..

\subsubsection{Methotrexate (MTX)}

To overcome the drug resistance of P-glycoprotein, multiple clinical trials were conducted. We report a case of a 69-year-old man with NK cell lymphoblastic leukemia /lymphoma who was treated mainly with methotrexate (MTX) (Kobayashi, 2010). He presented with skin and bone marrow involvement at onset. Neoplastic cells were blastic in appearance with a CD3-, CD4-, CD8-, CD7-, CD16-, CD56+ and HLA-DR+ phenotype. Molecular studies showed germline configuration of both immunoglobulin $\mathrm{JH}$ and $\mathrm{T}$ cell receptor genes and negative results for EBER. He was treated with standard ALL-targeting induction therapy, followed by one cycle of high-dose MTX(HD-MTX) as consolidation therapy. However, the disease recurred during the standard ALL maintenance therapy. Three cycles of HD-MTX were effective in achieving a second complete remission and he then received low dose MTX as maintenance therapy. The disease remained well-controlled for 4 years. MTX is one component of SMILE (methotrexate, ifosfamide, dexamethasone, etoposide, L-asparaginase) therapy. This encouraging result warrants further investigation of MTX either as a single agent or in a combination regimen as a first-line treatment for patients with NK cell malignancies.

\subsubsection{Combination chemotherapy: SMILE}

Japanese investigators treated advanced extranodal NK lymphoma with SMILE (Methotrexate, Ifosfamide, Dexamethasone, Etoposide, L-asparaginase) therapy(Yamaguchi, 2008). For newly diagnosed stage IV or relapsed/refractory ENKL, the overall response rate was $74 \%$ and the CR rate was $40 \%$. 
Another study with patients had newly diagnosed stage IV, relapsed or refractory diseases after first-line chemotherapy, were 15-69 years of age, and had satisfactory performance scores (0-2) (Jaccard, 2011). For the six enrolled patients, the overall response rate was $67 \%$ and the complete response rate was $50 \%$. Its safety and efficacy require further evaluation.

To explore an effective salvage regimen, phase I pilot study of combination chemotherapy with methotrexate, ifosfamide, l-asparaginase and dexamethasone (MILD), which are unaffected by MDR1-encoded P-glycoprotein, was tested in 18 patients(Tsukune, 2010). Among them, eleven had T/NK-cell malignancies, six had B-cell malignancies, and one had a blastic plasmacytoid dendritic cell neoplasm. Of the 14 patients evaluated, three achieved $\mathrm{CR}$, and four showed a partial response(PR). The overall response rate was $57 \%$. All seven responders had T/NK-cell malignancies. MILD therapy was feasible and presented acceptable toxicity in patients with refractory or lethal lymphoid malignancies.

\subsection{Hematopoietic Stem Cell Transplantation (HSCT)}

Because of the poor prognosis, many groups have favored the use of early transplantation of NK-cel neoplasms (Murashige. 2005:Suzuki, 2006; Ennishi, 2011; Ichikawa, 2010).

Autologous HSCT has been evaluated in stage I/II disease in first or second CR, or chemosensitive relapse, and primary or secondary refractory disease without marrow involvement. The disease status pre-HSCT significantly affected overall survival. For most patients with stage I/II disease, the definite advantage of auto HSCT in first CR is questionable. However, based on a retrospective analysis, auto HSCT may be beneficial in a subgroup of patients in CR1 who have a high risk of relapse. HSCT is generally indicated in lymphoma patients achieving second $\mathrm{CR}$, however further controlled trials are required to examine whether this also applies to NK/T-cell lymphoma. There is no survival advantage of auto HSCT in patients with advanced or refractory disease. Allogeneic HSCT, with the potential benefit of graft-versus-lymphoma (GVL) effect, is an option for patients with advanced disease. The GVL effect is further enhanced by the expression of EBV antigen on tumor cells. Small series have shown that it is a potentially curative option.

The retrospective analysis studied the potential survival benefits of HSCT for ENKL compared with a historical control group. Forty-seven patients from 3 previously published series of HSCT were matched according to NK/T cell lymphoma International Prognostic Index (NK-IPI) risk groups and disease status at transplantation. After a median follow-up of 116.5 months, the median survival time was not determined for the HSCT group, but it was 43.5 months for the control group. In patients who were in CR at the time of HSCT or at surveillance after remission, disease-specific survival rates were significantly higher in the HSCT group compared with the control group (disease-specific 5-year survival rate, $87.3 \%$ for HSCT vs $67.8 \%$ for non-HSCT). In contrast, in subgroup analysis on non-CR patients at the time of HSCT or non-HSCT treatment, disease-specific survival rates were not significantly prolonged in the HSCT group compared with the control group (1-year survival rate, $66.7 \%$ for HSCT vs $28.6 \%$ for non-HSCT). The impact of HSCT on the survival of all patients was significantly retained at the multivariate level with a 2.1-fold reduced risk of death. HSCT seems to confer a survival benefit in patients who attained CR on postremission consolidation therapy. These findings suggest that patients in CR with high NKIPI risk scores at diagnosis should receive full consideration for HSCT.

Among NK neoplasm, ANKL is a highly aggressive lymphoproliferative disease. a few case reports have suggested that allo-HSCT can be curative. For example, a report of a young 
woman with ANKL showing central nervous system invasion, who has been in complete remission for more than a year after allo-HSCT following two courses of intravenous chemotherapy and several rounds of intrathecal chemotherapy(Ichikawa, 2010).

However, most of the reported cases have been performed from HLA-identical sibling donors, and data on alternative HSCT sources including matched unrelated donor and umbilical cord blood are very limited.

\subsection{Radiation}

For localized diseases, radiation therapy is reportedly effective. For example, the addition of radiotherapy for early-stage nasal NK cell lymphoma cases yielded survival benefit (P = .045)(Suzuki. 2010).

\subsection{New therapeutic strategies}

\subsubsection{Bortezomib}

Basic studies showed that LMP1 activates nuclear factor-kB (NF-kB) and that NF-kB is activated in extranodal natural killer (NK)/T-cell lymphoma, nasal type(Shen, 2008). Bortezomib is known to be effective for diseases involving NF-kB activation, such as multiple myeloma. Recently, bortezomib was reported to induce apoptosis of EBVtransformed B cells.

Twenty three patients with previously untreated NK/T-cell lymphoma initially treated with cyclophosphamide, vincristine, doxorubicin and prednisone (CHOP) or CHOP-based chemotherapy were examined by immunohistochemistry for three NF-kB subunits(p65, p50 and p52), which are involved in either the canonical or alternative pathway. NF-KB activation through the alternative pathway is frequently observed in NK/T-cell lymphoma and associated with chemoresistance and poor survival. In vitro, bortezomib treatment decreased the viability of NK cell lines. The decreased viability in response to bortezomib treatment was abrogated by a pan-caspase inhibitor. Additionally, cleavage of caspases and polyadenosine diphosphate -ribose polymerase, increased expression of phosphorylated $\mathrm{I \kappa B}$, and decreased expression of inhibitor of apoptotic proteins were detected by immunoblotting in bortezomib-treated cell lines. Administration of bortezomib to peripheral blood mononuclear cells from two patients with EBV-associated lymphoproliferative diseases show a greater killing effect on EBV-infected cells. These results indicate that bortezomib killed T or NK lymphoma cells by inducing apoptosis.

A Phase I study of Bortezomib + CHOP treatment were conducted with 13 patients with advanced, aggressive T-cell or NK/T-cell lymphoma(Lee, 2008). The overall CR rate in all patients was $61.5 \%$ and showed no severe side effects. Bortezomib can be safely combined with $\mathrm{CHOP}$ chemotherapy and constitutes an active regimen.

\subsubsection{Pegaspargase}

Pegaspargase selected the pegylated form to achieve more prolonged continuous asparagine depletion as well as for ease of administration as it requires only a single treatment every 2-3 weeks(Reyes, 2008). Pegaspargase were reported an effective treatment for two patients with aggressive, extended, and refractory to CHOP chemotherapy. Pegaspargase is worth considering development of combination regimens and possibly front-line regimens. 


\subsubsection{Kinase inhibitor}

The EBV oncoprotein LMP1 reportedly activates the phosphatidylinositol-3 kinase (PI3K)/Akt pathway to induce cell survival(Jeon, 2007). The intrinsic level of pAkt was higher in EBV-positive NK cells than in EBV-negative NK cells. Geldanamycin (GA) and its derivative, 17-allylamino-17- demethoxygeldanamycin (17-AAG), are PI3K and Akt inhibitors that exhibit anti-tumor activity by degrading HSP90 client proteins, including Akt. The administration of GA and 17-AAG resulted in apoptosis of NK cells, accompanied by Akt and pAkt down-regulation, caspase 3 activation, and mitochondrial membrane potential disruption. Apoptosis of NK cells was also induced by LY294002 (a PI3K inhibitor) or Akt inhibitor II. More results both in vitro and in vivo are necessary to determine the efficacy of kinase inhibitors for treating NK neoplasms.

\subsubsection{Antibody treatment}

The immunoconjugate I MGN901 (huN901-DM1; ImmunoGen, Cambridge, MA,USA) is composed of the composed of the humanized monoclonal IgG1 antibody, huN901, and the maytansinoid drug, DM1, which binds CD56 with high affinity(Ishitsuka, 2009). IMGN901 is a tumour-activated prodrug because the conjugation of DM1 to huN901 renders the cytotoxic drug inactive until it reaches the target site. The conjugate is then internalized and releases DM1, which inhibits tubulin polymerization and causes cell death. IMGN901 has been demonstrated to be safe and show promising efficacy in small cell lung cancer, CD56positive small cell carcinoma and multiple myeloma in phase I/II clinical studies. An in vitro study demonstrated that IMGN901 was cytotoxic for a CD56-positive NK cell line and fresh tumour cells derived from a patient with an NK-cell malignancy in vitro. These results indicate that IMGN901 represents a promising novel and targeted approach to improve patient outcomes for patients with NK cell malignancies. The activity of IMGN901 should be further validated in clinical trials.

\section{Conclusion}

The classification of NK cell neoplasms with overlapping features will remain controversial, particularly when EBV is absent. In order to establish more definitive and widely accepted diagnostic criteria for NK-cell neoplasms, more accurate diagnostic tools are needed. For proper treatment stratification of NK cell neoplasms, randomized clinical trials are waited, although conducting meaningful clinical trials is difficult because NK-cell neoplasms are rare.

\section{References}

$\mathrm{Au}$ WY, Weisenburger DD, Intragumtornchai T, et al.; International Peripheral T-Cell Lymphoma Project.(2009) Clinical differences between nasal and extranasal natural killer/T-cell lymphoma: a study of 136 cases from the International Peripheral TCell Lymphoma Project. Blood. Vol 113. No 17. pp.3931-7.

Dellis O, Arbabian A, Brouland JP, et al.(2009) Modulation of B-cell endoplasmic reticulum calcium homeostasis by Epstein-Barr virus latent membrane protein-1. Mol Cancer. Vol.8, pp.59-73.

Egashira M, Kawamata N, Sugimoto K, et al.(1993) P-glycoprotein expression on normal and abnormally expanded natural killer cells and inhibition of P-glycoprotein function by cyclosporin A and its analogue, PSC833. Blood. Vol. 93. No. 5. pp.599-606 
Ennishi D, Maeda Y, Fujii N, et a;.(2011) Allogeneic hematopoietic stem cell transplantation for advanced extranodal natural killer/T-cell lymphoma, nasal type. Leuk Lymphoma. 2011 May 23. [Epub ahead of print]

Guan XQ, Xu L, Ke ZY, et.al(2011) Five Chinese pediatric patients with leukemias possibly arising from immature natural killer cells: clinical features and courses. Pediatr Hematol Oncol. Vol 28. No.3. pp187-93.

Grzywacz B, (2011) Natural killer-cell differentiation by myeloid progenitors. Blood. Vol.117, No.13, pp. 3548-58.

Ham MF,\& Ko YH.(2010) Natural killer cell neoplasm: biology and pathology. Int J Hematol. Vol.92, No.5. pp.681-9.

Hsieh PP, Tung CL, Chan AB, et al.(2007) EBV viral load in tumor tissue is an important prognostic indicator for nasal NK/T-cell lymphoma. Am J Clin Pathol. Vol 128. No.4. pp.579-84.

Huang WT, Chang KC, Huang GC, et al.(2005) Bone marrow that is positive for Epstein-Barr virus encoded RNA-1 by in situ hybridization is related with a poor prognosis in patients with extranodal natural killer/T-cell lymphoma, nasal type. Haematologica. Vol. 90.No. 8. pp.1063-9.

Huang Y, de Reyniès A, de Leval L, et al.(2010) Gene expression profiling identifies emerging oncogenic pathways operating in extranodal NK/T-cell lymphoma, nasal type. Blood. Vol 115. No.6. pp1226-37.

Ian MX, Lan SZ, Cheng ZF, et.al(2008) Suppression of EBNA1 expression inhibits growth of EBV-positive NK/T cell lymphoma cells. Cancer Biol Ther. Vol 7. No.10. pp1602-6.

Ichikawa S, Fukuhara N, Yamamoto J, , et al.(2010) Successful allogeneic hematopoietic stem cell transplantation for aggressive NK cell leukemia. Intern Med. Vol 49, No.17. pp.1907-10.

Ishitsuka K, Jimi S, Goldmacher VS, et al.(2009) Targeting CD56 by the maytansinoid immunoconjugate IMGN901 (huN901-DM1): a potential therapeutic modality implication against natural killer/T cell malignancy. Blood. Vol 114. No.15. pp.3265-75.

Jaccard A, Petit B, Girault S, et al.(2009) L-asparaginase-based treatment of 15 western patients with extranodal NK/T-cell lymphoma and leukemia and a review of the literature. Ann Oncol. Vol 20. No.1, pp.110-6.

Jaccard A, Gachard N, Marin B, et al; GELA and GOELAMS Intergroup.(2011) Efficacy of Lasparaginase with methotrexate and dexamethasone (AspaMetDex regimen) in patients with refractory or relapsing extranodal NK/T-cell lymphoma, a phase 2 study.Blood. Vol 117. No.6. pp.1834-9.

Jeon YK, Park CH, Kim KY, et al.(2007) The heat-shock protein 90 inhibitor, geldanamycin, induces apoptotic cell death in Epstein-Barr virus-positive NK/T-cell lymphoma by Akt down-regulation.J Pathol. Vol 213, No.2, pp.170-9.

Kobayashi S, Teramura M, Arai M, et al.(2010) Successful salvage treatment of blastic natural killer cell lymphoma with methotrexate. Int J Hematol. Vol.92, No.4, pp.634-7.

Lee J, Suh C, Park YH, , et al.(2006) Extranodal natural killer T-cell lymphoma, nasal-type: a prognostic model from a retrospective multicenter study. J Clin Oncol. Vol. 24, No.4. pp.612-8.

Lee J, Suh C, Kang HJ, et al.(2008) Phase I study of proteasome inhibitor bortezomib plus $\mathrm{CHOP}$ in patients with advanced, aggressive T-cell or NK/T-cell lymphoma. Ann Oncol. Vol 19. No.12, pp.2079-83. 
Mansoor A, Pittaluga S, Beck PL, et.al(2011) NK-cell enteropathy: a benign NK-cell lymphoproliferative disease mimicking intestinal lymphoma: clinicopathologic features and follow-up in a unique case series. Blood. Vol.117. No.5. pp1447-52.

Murashige N, Kami M, Kishi Y, et al.(2005) Allogeneic haematopoietic stem cell transplantation as a promising treatment for natural killer-cell neoplasms. $\mathrm{Br} \mathrm{J}$ Haematol. Vol.130, No.4. pp.561-7.

Ng SB, Selvarajan V, Huang G, Zhou J, Feldman AL, Law M, et al.(2011) Activated oncogenic pathways and therapeutic targets in extranodal nasal-type NK/T cell lymphoma revealed by gene expression profiling.J Pathol. Vol 223. No.4. pp.496-510.

Oshimi K, Kawa K, Nakamura S, et al; NK-cell Tumor Study Group.(2005) NK-cell neoplasms in Japan.Hematology. Vol.10, No.3, pp.237-45.

Oshimi K.(2007) Progress in understanding and managing natural killer-cell malignancies. Br J Haematol. Vol.139, No.4, pp.532-44.

Pilichowska ME, Fleming MD, Pinkus JL, et.al.(2007) CD4+/CD56+ hematodermic neoplasm ("blastic natural killer cell lymphoma"): neoplastic cells express the immature dendritic cell marker BDCA-2 and produce interferon. Am J Clin Pathol. Vol 128, No.3. pp445-53.

Pizzigallo E, Racciatti D, and Gorgoretti V(2010) EBV Chronic Infections Mediterr J Hematol Infect Dis. Vol.2. No.1:e2010022.

Reyes VE Jr, Al-Saleem T, Robu VG, et al.(2008) Extranodal NK/T-cell lymphoma nasal type: efficacy of pegaspargase. Report of two patients from the United Sates and review of literature. Br J Haematol. Vol.141, No.1. pp.129-31.

Schmitt C, Sako N, Bagot M et.al.(2011) Extranodal NK/T-Cell Lymphoma: Toward the Identification of Clinical Molecular Targets. J Biomed Biotechnol. :790871.

Shen L, Au WY, Wong KY, et al.(2008) Cell death by bortezomib-induced mitotic catastrophe in natural killer lymphoma cells. Mol Cancer Ther. Vol 7. No.12. pp.3807-15.

Shiba N, Kanazawa T, Park MJ, et al.(2010) NOTCH1 mutation in a female with myeloid/NK cell precursor acute leukemia. Pediatr Blood Cancer. Vol 55. No.7. pp1406-9.

Sun, JC.(2010) Re-educating natural killer cells. J Exp Med. Vol.207, No.10, pp.2049-52.

Suzuki R, Yamamoto K, Seto M, et al.(1997) CD7+ and CD56+ myeloid/natural killer cell precursor acute leukemia: a distinct hematolymphoid disease entity. Blood. Vol 90. No.6. pp.2417-28.

Suzuki R, Suzumiya J, Oshimi K.(2009) Differences between nasal and extranasal NK/T-cell lymphoma. Blood. 113(17):3931-7.

Suzuki R, Ohtake S, Takeuchi J, et al.(2010) The clinical characteristics of CD7+ CD56+ acute myeloid leukemias other than M0. Int J Hematol. Vol.91, No.2.pp.303-9.

Suzuki R, Nakamura S, Suzumiya J, et al.(2005) Blastic natural killer cell lymphoma/leukemia (CD56-positive blastic tumor). Cancer Vol.104, No.5,pp.1022-31

Suzuki R, Suzumiya J, Nakamura S, et al. NK-cell Tumor Study Group.(2006) Hematopoietic stem cell transplantation for natural killer-cell lineage neoplasms. Bone Marrow Transplant. Vol.37. No.4, pp.425-31.

Suzuki R, Suzumiya J, Yamaguchi M, et al.(2010); NK-cell Tumor Study Group. Prognostic factors for mature natural killer (NK) cell neoplasms: aggressive NK cell leukemia and extranodal NK cell lymphoma, nasal type.

Suzuki R.(2010) Treatment of advanced extranodal NK/T cell lymphoma, nasal-type and aggressive NK-cell leukemia.Int J Hematol. Vol.92.No.5. pp.697-701. 
Swerdlow SH, et al. (2008) WHO classification of tumours of haematopoietic and lymphoid tissues. Fourth edition, Lyon, IARC Press. Ann Oncol. Vol.21. No.5. pp.1032-40.

Takeuchi K, Yokoyama M, Ishizawa S, et al. Lymphomatoid gastropathy: a distinct clinicopathologic entity of self-limited pseudomalignant NK-cell proliferation. Blood. Vol.116, No.25. pp.5631-7.

Tang G, Truong F, Fadare O. et.al (2008) Diagnostic challenges related to myeloid/natural killer cells, a variant of myeloblasts. Int J Clin Exp Pathol. Vol 1. No.6. pp544-9.

Tsukune Y, Isobe Y, Yasuda H, et al.(2010) Activity and safety of combination chemotherapy with methotrexate, ifosfamide, l-asparaginase and dexamethasone (MILD) for refractory lymphoid malignancies: a pilot study.Eur J Haematol. Vol.84. No.4. pp.310-5.

Yamaguchi M, Suzuki R, Kwong YL, et al.(2008) Phase I study of dexamethasone, methotrexate, ifosfamide, L-asparaginase, and etoposide (SMILE) chemotherapy for advanced-stage, relapsed or refractory extranodal natural killer (NK)/T-cell lymphoma and leukemia.Cancer Sci. Vol 99. No.5. pp.1016-20.

Yok-Lam K.(2011) The Diagnosis and Management of Extranodal NK/T-Cell Lymphoma, Nasal-Type and Aggressive NK-Cell Leukemia.J Clin Exp Hematop. Vol.51, No.1, pp.21-8.

Yong W, Zheng W, Zhu J, Zhang Y, Wei Y, Wang X, et al.(2006) Midline NK/T-cell lymphoma nasal-type: treatment outcome, the effect of L-asparaginase based regimen, and prognostic factors.Hematol Oncol. Vol 24, No.1. pp.28-32.

Zhang L, Anglesio MS, O'Sullivan M, Zhang F, Yang G, Sarao R, et al.(2007) The E3 ligase HACE1 is a critical chromosome 6q21 tumor suppressor involved in multiple cancers.Nat Med. Vol.13. No.9. pp1060-9. 


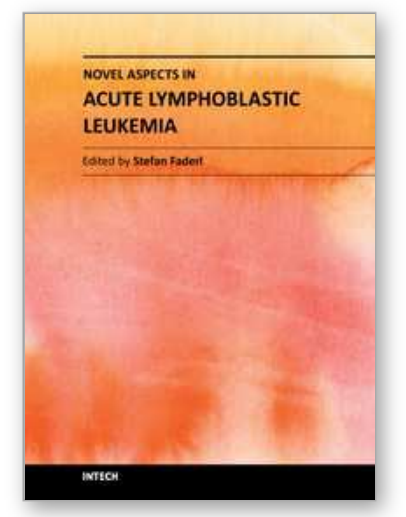

\author{
Novel Aspects in Acute Lymphoblastic Leukemia \\ Edited by Dr. Stefan Faderl
}

ISBN 978-953-307-753-6

Hard cover, 258 pages

Publisher InTech

Published online 16, November, 2011

Published in print edition November, 2011

Acute lymphoblastic leukemia (ALL) has turned from a universally fatal to a highly curable disease in little more than four decades. Even though differences in outcome continue to exist between children and adults, intense efforts are under way to overcome this discrepancy and improve the prognosis of adult patients as well. This exemplary progress in ALL therapy has been possible by the combination of an increasingly better understanding of the biology of the disease, availability of a range of effective drugs, and astute designs and relentless executions of many clinical trials. ALL is a complex disease requiring complex therapy. Whereas this book cannot provide a comprehensive review of every one of its many facets, the chapters from many investigators from around the world nevertheless cover a number of relevant topics: aspects of the epidemiology of ALL in Hispanics, ophthalmologic manifestations of ALL, overviews of current therapy and drug-resistance mechanisms, novel biological pathways and targets, new drugs in development, and long-term consequences of CNS prophylaxis and therapy. The publishers and editor therefore hope that the prospective readers will find enough insight and information for their own endeavors.

\title{
How to reference
}

In order to correctly reference this scholarly work, feel free to copy and paste the following:

Shoko Kobayashi (2011). Natural Killer Cell Leukemia: Diagnosis, Pathogenesis and Treatment, Novel Aspects in Acute Lymphoblastic Leukemia, Dr. Stefan Faderl (Ed.), ISBN: 978-953-307-753-6, InTech, Available from: http://www.intechopen.com/books/novel-aspects-in-acute-lymphoblastic-leukemia/natural-killer-cell-leukemiadiagnosis-pathogenesis-and-treatment

\section{INTECH}

open science | open minds

\section{InTech Europe}

University Campus STeP Ri

Slavka Krautzeka 83/A

51000 Rijeka, Croatia

Phone: +385 (51) 770447

Fax: +385 (51) 686166

www.intechopen.com

\section{InTech China}

Unit 405, Office Block, Hotel Equatorial Shanghai

No.65, Yan An Road (West), Shanghai, 200040, China

中国上海市延安西路65号上海国际贵都大饭店办公楼405单元

Phone: +86-21-62489820

Fax: +86-21-62489821 
(C) 2011 The Author(s). Licensee IntechOpen. This is an open access article distributed under the terms of the Creative Commons Attribution 3.0 License, which permits unrestricted use, distribution, and reproduction in any medium, provided the original work is properly cited. 